\title{
Nocardioides salarius sp. nov., isolated from seawater enriched with zooplankton
}

\author{
Correspondence \\ Byung Cheol Cho \\ bccho@snu.ac.kr
}

\author{
Hye Min Kim, ${ }^{1}$ Dong Han Choi, ${ }^{2}$ Chung Yeon Hwang ${ }^{1}$ \\ and Byung Cheol $\mathrm{Cho}^{1}$
}

\author{
${ }^{1}$ School of Earth and Environmental Sciences, Seoul National University, Shillim-dong, Kwanak-gu, \\ Seoul 151-742, Republic of Korea \\ ${ }^{2}$ Marine Environmental Research Department, Korea Ocean Research and Development Institute, \\ Ansan 426-744, Republic of Korea
}

\begin{abstract}
A rod-shaped marine bacterium, designated $\mathrm{CL}-\mathrm{Z} 59^{\top}$, was isolated from seawater enriched with zooplankton. Phylogenetic analyses based on 16S rRNA gene sequences showed that strain CL$\mathrm{Z}_{59}{ }^{\top}$ belongs to the genus Nocardioides, the highest level of sequence similarity (99.2\%) being found with respect to Nocardioides marinisabuli KCCM $42681^{\top}$. However, DNA-DNA hybridization experiments between strain CL-Z59 ${ }^{\top}$ and $N$. marinisabuli $\mathrm{KCCM} 42681^{\top}$ revealed a mean relatedness value of $33 \%$. Cells were Gram-positive, strictly aerobic and non-motile. Strain $\mathrm{CL}-\mathrm{Z59}{ }^{\top}$ grew optimally at $\mathrm{pH} 6-7$ and $25-30{ }^{\circ} \mathrm{C}$ and at a $\mathrm{NaCl}$ concentration of $3 \%$. The isolate was characterized chemotaxonomically as having LL-diaminopimelic acid in the cell wall. The major isoprenoid quinone was $\mathrm{MK}-8\left(\mathrm{H}_{4}\right)$ and the predominant cellular fatty acid was iso$\mathrm{C}_{16: 0}$. The DNA G $+\mathrm{C}$ content was $73.3 \mathrm{~mol} \%$. On the basis of phylogenetic, genotypic and phenotypic data, strain CL-Z59 ${ }^{\top}$ represents a novel species of the genus Nocardioides, for which the name Nocardioides salarius sp. nov. is proposed. The type strain is CL-Z59 ${ }^{\top}$ (=KCCM $42320^{\top}=$ DSM $18239^{\top}$ ).
\end{abstract}

At the time of writing, since the description of the genus Nocardioides within the class Actinobacteria (Prauser, 1976), 27 species of the genus have been isolated from various environments. Nocardioides simplex (Jensen, 1934), Nocardioides albus (Prauser, 1976), Nocardioides jensenii (Suzuki \& Komagata, 1983; Collins et al., 1989), Nocardioides luteus (Prauser, 1984), Nocardioides alkalitolerans (Yoon et al., 2005a), Nocardioides kribbensis (Yoon et al., 2005b), Nocardioides dubius (Yoon et al., 2005c), Nocardioides lentus (Yoon et al., 2006a), Nocardioides kongjuensis (Yoon et al., 2006b), Nocardioides insulae (Yoon et al., 2007a), Nocardioides panacihumi (An et al., 2007), Nocardioides hankookensis (Yoon et al., 2008) and Nocardioides terrigena (Yoon et al., 2007b) were isolated from various soils. Nocardioides pyridinolyticus (Yoon et al., 1997) and Nocardioides oleivorans (Schippers et al., 2005) were isolated from the oxic zone of an oil shale column and crude oil, respectively. In addition, Nocardioides plantarum (Collins et al., 1994) was isolated from herbage, and Nocardioides nitrophenolicus (Yoon et al., 1999), Nocardioides aquiterrae (Yoon et al., 2004) and Nocardioides aromaticivorans (Kubota et al., 2005) were

The GenBank/EMBL/DDBJ accession number for the 16S rRNA gene sequence of strain CL-Z59 ${ }^{\top}$ is DQ401092. isolated from non-saline aquatic environments (industrial wastewater, groundwater and a river, respectively). Nocardioides daphniae was isolated from a water flea originating from lake water (Tóth et al., 2008). Furthermore, seven species were isolated from saline environments: Nocardioides aquaticus (Lawson et al., 2000) was isolated from a saline lake in Antarctica, Nocardioides aestuarii (Yi \& Chun, 2004a) and Nocardioides ganghwensis (Yi \& Chun, 2004b) were isolated from tidal flats, Nocardioides furvisabuli (Lee, 2007), Nocardioides marinisabuli (Lee et al., 2007) and Nocardioides hwasunensis (Lee et al., 2008) were isolated from beach sand and Nocardioides marinus (Choi et al., 2007) was isolated from seawater. In this study, strain CL-Z59 ${ }^{\mathrm{T}}$ was isolated from seawater enriched with zooplankton and was subjected to a polyphasic taxonomic analysis.

To isolate zooplankton-associated bacteria, seawater was sampled from the surface of the South Sea, Korea, filtered using a syringe filter $(0.2 \mu \mathrm{m}$ pore size $)$ and dispensed into a $20 \mathrm{ml}$ autoclaved glass vial. Then the $0.2 \mu \mathrm{m}$-filtered seawater was supplemented with zooplankton [collected using a zooplankton net ( $200 \mu \mathrm{m}$ mesh size $)$ and rinsed with $0.2 \mu \mathrm{m}$ filter-sterilized seawater three times] and incubated at a temperature close to the in situ temperature 
(approx. $10-15{ }^{\circ} \mathrm{C}$ ). After about 1 year, $50 \mu \mathrm{l}$ aliquots were taken and spread on a plate of low-nutrient heterotrophic medium supplemented with $1 \times$ mixed carbons (Cho \& Giovannoni, 2004). After 1 month, each colony was streaked again on a plate of low-nutrient heterotrophic medium plus $1 \times$ mixed carbons, and subsequently on marine agar 2216 (MA; Difco). Strain CL-Z59 ${ }^{\mathrm{T}}$ grew better on MA and was routinely cultivated on MA at $30{ }^{\circ} \mathrm{C}$. The strain was maintained both on MA at $4{ }^{\circ} \mathrm{C}$ and in marine broth 2216 (Difco) supplemented with $30 \%$ (v/v) glycerol at $-80{ }^{\circ} \mathrm{C}$.

Morphological, physiological and biochemical analyses were performed. Unless otherwise specified, all characteristics described hereafter were based on cultures grown on MA at $30{ }^{\circ} \mathrm{C}$. Gram-staining was performed as described by Smibert \& Krieg (1994). The cell morphology and motility of the isolate were observed by using phase-contrast microscopy (BX50; Olympus). Transmission electron microscopy (EX2; JEOL) was used to establish whether flagella were present. Anaerobic growth was checked on MA and ZOF medium (Lemos et al., 1985) supplemented with additional agar (1.5\%), using the GasPak anaerobic system (BBL) at $30{ }^{\circ} \mathrm{C}$ for 15 days. Catalase and oxidase activities and decomposition of casein, hypoxanthine, xanthine and L-tyrosine were determined according to the protocols described by Smibert \& Krieg (1994). Nitrate reductase activity and degradation of starch and Tween 80 were investigated as described by Hansen \& Sørheim (1991).

The temperature range for growth was determined on the basis of the formation of single colonies visible on MA incubated at $5-45{ }^{\circ} \mathrm{C}$ (using increments of $5{ }^{\circ} \mathrm{C}$ ). The $\mathrm{pH}$ range (between $\mathrm{pH} 5$ and 10, using increments of $1 \mathrm{pH}$ unit) for growth was determined by assessing changes in the $\mathrm{OD}_{600}$ over time in marine broth 2216. The final $\mathrm{pH}$ was adjusted using $1 \mathrm{M} \mathrm{NaOH}$ and $1 \mathrm{M} \mathrm{HCl}$ solutions. The $\mathrm{NaCl}$ tolerance of strain $\mathrm{CL}-\mathrm{Z} 59^{\mathrm{T}}$ was determined using synthetic ZoBell broth (containing, $\mathrm{l}^{-1}$ distilled water: $5 \mathrm{~g}$ Bacto peptone, $1 \mathrm{~g}$ yeast extract, $0.1 \mathrm{~g}$ ferric citrate; Yi \& Chun, 2004b) with various $\mathrm{NaCl}$ concentrations $(0,0.1,0.3,0.5,1,2,3,4,5,6,7,8,9,10,15$ and $20 \%$, $\mathrm{w} / \mathrm{v})$. Nitrate reduction, indole production, arginine dihydrolase, urease, gelatinase and $\beta$-galactosidase, acid production from glucose and hydrolysis of aesculin were tested using an API 20NE kit (bioMérieux) according to the manufacturer's instructions, except that the cell suspension was prepared using artificial seawater at half strength (containing, $\mathrm{l}^{-1}$ distilled water: $12 \mathrm{~g} \mathrm{NaCl}, 5.44 \mathrm{~g}$ $\mathrm{MgCl}_{2} .6 \mathrm{H}_{2} \mathrm{O}, 2 \mathrm{~g} \mathrm{Na}_{2} \mathrm{SO}_{4}, 0.73 \mathrm{~g} \mathrm{CaCl}_{2} .2 \mathrm{H}_{2} \mathrm{O}, 0.35 \mathrm{~g}$ $\mathrm{KCl}, 0.1 \mathrm{~g} \mathrm{NaHCO}_{3}, 0.05 \mathrm{~g} \mathrm{KBr}, 0.0135 \mathrm{~g} \mathrm{H}_{3} \mathrm{BO}_{3}, 0.02 \mathrm{~g}$ $\mathrm{SrCl}_{2} .6 \mathrm{H}_{2} \mathrm{O}$ and $0.015 \mathrm{~g} \mathrm{NaF}$; Lyman \& Fleming, 1940) as the suspension medium. Other enzymic activities were determined using an API ZYM kit (bioMérieux) and halfstrength artificial seawater as the suspension medium. Carbon utilization was tested using a modified basal broth medium (containing, $1^{-1}$ distilled water: $11.8 \mathrm{~g} \mathrm{NaCl}$, $0.32 \mathrm{~g} \mathrm{KCl}, 2.26 \mathrm{~g} \mathrm{MgCl}_{2} .6 \mathrm{H}_{2} \mathrm{O}, 2.97 \mathrm{~g} \mathrm{MgSO}_{4} \cdot 7 \mathrm{H}_{2} \mathrm{O}$,
$0.65 \mathrm{~g} \mathrm{CaCl}_{2} .2 \mathrm{H}_{2} \mathrm{O}, 0.2 \mathrm{~g} \mathrm{NaNO}_{3}, 0.2 \mathrm{~g} \mathrm{NH}_{4} \mathrm{Cl}$ and $0.05 \mathrm{~g}$ yeast extract; $\mathrm{pH} 6.7 \pm 0.5$ ) containing $0.2 \%$ carbon source. Carbon utilization was scored as negative when growth was equal to, or less than, that in the negative control with no carbon source. Growth was measured by monitoring changes in the $\mathrm{OD}_{600}$ after 8-12 days incubation at $30{ }^{\circ} \mathrm{C}$.

Isoprenoid quinones were isolated according to the method of Minnikin et al. (1984) and were analysed by HPLC as described by Collins (1985) at the Korean Culture Center of Microorganisms, Seoul, Korea. The cell-wall diamino acids were determined as described by Staneck \& Roberts (1974). The whole-cell fatty acid methyl esters in strain CL-Z59 ${ }^{\mathrm{T}}$, grown on MA at $30{ }^{\circ} \mathrm{C}$ for 1 day, were analysed at the Korean Culture Center of Microorganisms, using gas chromatography according to the instructions of the Microbial Identification System (MIDI). Genomic DNA-DNA relatedness was determined by using dot-blot hybridization (Kim et al., 2007). Prehybridization, hybridization and detection were performed using a DIG labelling and detection kit (Roche Molecular Biochemicals) according to the manufacturer's instructions. The experiment was repeated on different days.

For 16S rRNA gene amplification with PCR, DNA was extracted from a single colony by using a boiling method (Englen \& Kelley, 2000). The crude extracts served as the DNA template for the PCR reactions, which involved Taq DNA polymerase (Bioneer) and primers 27F and 1492R (Lane, 1991). The PCR product was purified by using an AccuPrep PCR purification kit (Bioneer) and was cloned using the pGEM-T Easy vector (Promega). Sequencing of the 16S rRNA gene was performed with an Applied Biosystems automated sequencer (ABI3730XL) at Macrogen Corp. (Seoul, Korea). An almost-complete 16S rRNA gene sequence (1446 bp) of strain CL-Z59 ${ }^{\mathrm{T}}$ was obtained. The sequence was compared with those available in the GenBank database by using BLASTN searches (Altschul et al., 1990). The 16S rRNA gene sequence of strain CL-Z59 ${ }^{\mathrm{T}}$ was manually aligned with those of the type strains of other species belonging to the genus Nocardioides and with phylogenetically related genera obtained from GenBank and the Ribosomal Database Project (Cole et al., 2003) database by using known 16S rRNA gene secondary-structure information. Phylogenetic trees were obtained by using the neighbourjoining (Saitou \& Nei, 1987) and maximum-parsimony (Fitch, 1971) methods. An evolutionary distance matrix for the neighbour-joining method was generated according to the model of Jukes \& Cantor (1969). The robustness of tree topologies was assessed by using bootstrap analyses based on 1000 replications for the neighbour-joining and maximum-parsimony methods. Alignment analysis was carried out using the jPHYDIT program (Jeon et al., 2005) and phylogenetic analyses were carried out using MEGA3 (Kumar et al., 2004). The DNA $\mathrm{G}+\mathrm{C}$ content was determined at the Korean 
Table 1. Characteristics that serve to differentiate strain CL-Z59 $9^{\top}$ from related Nocardioides species

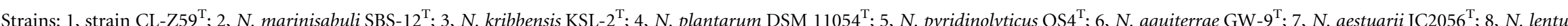

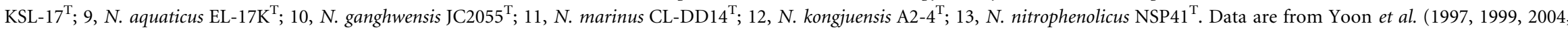

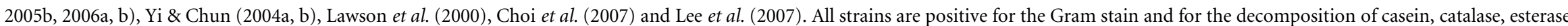

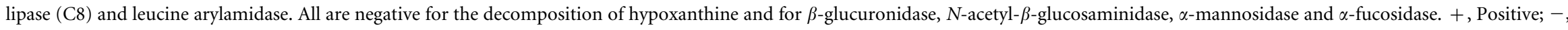
negative; $\mathrm{W}$, weakly positive; $\mathrm{V}$, variable; ND, not determined.

\begin{tabular}{|c|c|c|c|c|c|c|c|c|c|c|c|c|c|}
\hline Characteristic & 1 & 2 & 3 & 4 & 5 & 6 & 7 & 8 & 9 & 10 & 11 & 12 & 13 \\
\hline Cell morphology & Rods & Rods & $\begin{array}{l}\text { Short rods, } \\
\text { cocci }\end{array}$ & $\begin{array}{l}\text { Short rods, } \\
\text { cocci }\end{array}$ & $\begin{array}{l}\text { Rods, } \\
\text { cocci }\end{array}$ & $\begin{array}{l}\text { Rods, } \\
\text { cocci }\end{array}$ & Rods & $\begin{array}{l}\text { Rods, } \\
\text { cocci }\end{array}$ & $\begin{array}{l}\text { Cocci, short } \\
\text { rods }\end{array}$ & Rods & $\begin{array}{l}\text { Rods, } \\
\text { cocci }\end{array}$ & $\begin{array}{l}\text { Rods, } \\
\text { cocci }\end{array}$ & Rods \\
\hline Cell size $(\mu \mathrm{m})$ & $\begin{array}{c}0.3- \\
0.6 \times 0.6- \\
1.6\end{array}$ & $\begin{array}{c}0.6- \\
0.8 \times 1.4- \\
2.1\end{array}$ & $\begin{array}{c}0.8- \\
1.0 \times 1.5- \\
2.0\end{array}$ & ND & $\begin{array}{c}0.5- \\
0.6 \times 1.2- \\
1.6\end{array}$ & $\begin{array}{c}0.8- \\
1.0 \times 1.7- \\
2.0\end{array}$ & $\begin{array}{c}0.3- \\
0.4 \times 0.9- \\
2.1\end{array}$ & $\begin{array}{c}0.4- \\
0.7 \times 1.0- \\
4.5\end{array}$ & $\begin{array}{c}0.9- \\
1.0 \times 0.9- \\
1.4\end{array}$ & $\begin{array}{c}0.4- \\
0.5 \times 0.9- \\
4.5\end{array}$ & $\begin{array}{c}0.4- \\
0.6 \times 1.0- \\
1.8\end{array}$ & $\begin{array}{c}0.4- \\
0.7 \times 0.8- \\
3.0\end{array}$ & $\begin{array}{c}0.5- \\
0.8 \times 1.0- \\
3.0\end{array}$ \\
\hline Colony colour & Cream & Pale yellow & Cream & ND & Cream & Cream & Ivory & Yellow & Dull orange & Ivory & Cream & $\begin{array}{l}\text { Yellowish } \\
\text { white }\end{array}$ & $\begin{array}{c}\text { Yellowish } \\
\text { white }\end{array}$ \\
\hline $\begin{array}{l}\text { pH range } \\
\text { (optimum) }\end{array}$ & $\begin{array}{l}6-10 \\
(6-7)\end{array}$ & $\begin{array}{l}5.1-12.1 \\
(7.1-8.1)\end{array}$ & $\begin{array}{r}6-11 \\
(9)\end{array}$ & $\mathrm{ND}$ & $\begin{array}{r}5-9 \\
(8)\end{array}$ & $(6-7)$ & $\begin{array}{r}6-10 \\
(7)\end{array}$ & $\begin{array}{c}6.5-9.5 \\
\quad(8)\end{array}$ & $\begin{array}{r}5.5-9.5 \\
(7-8)\end{array}$ & $\begin{array}{r}6-10 \\
(7)\end{array}$ & $\begin{array}{l}6-9 \\
(7-8)\end{array}$ & $\begin{array}{r}5.5-10 \\
(7-8)\end{array}$ & $\begin{array}{r}6-10 \\
(8)\end{array}$ \\
\hline $\begin{array}{l}\text { Temperature range } \\
\text { (optimum) }\left({ }^{\circ} \mathrm{C}\right)\end{array}$ & $\begin{array}{l}10-35 \\
(25-30)\end{array}$ & $4-40$ & $\begin{array}{r}4-35 \\
(30)\end{array}$ & 25 & $\begin{array}{r}20-40 \\
(35)\end{array}$ & $\begin{array}{c}15-42 \\
(30)\end{array}$ & $\begin{array}{r}20-35 \\
(30)\end{array}$ & $\begin{array}{r}4-34 \\
(28)\end{array}$ & $\begin{array}{l}3-43.5 \\
(16-26)\end{array}$ & $\begin{array}{r}10-40 \\
(30)\end{array}$ & $\begin{array}{l}10-40 \\
(25-30)\end{array}$ & $\begin{array}{l}10-40 \\
(30-37)\end{array}$ & $\begin{array}{c}15-40 \\
(30)\end{array}$ \\
\hline $\begin{array}{l}\mathrm{NaCl} \text { range } \\
\text { (optimum) (\%) }\end{array}$ & $\begin{array}{r}1-10 \\
(3)\end{array}$ & $0-8$ & $0-3$ & ND & $\mathrm{ND}$ & $\mathrm{ND}$ & $\begin{array}{l}0-8 \\
(0-2)\end{array}$ & $\begin{array}{l}0-5 \\
(0.5)\end{array}$ & $\begin{array}{l}0-15 \\
(1-6)\end{array}$ & $\begin{array}{l}0-8 \\
(0-1)\end{array}$ & $\begin{array}{c}0.5-8 \\
(1-3)\end{array}$ & $\begin{array}{r}0-5 \\
(0)\end{array}$ & $\mathrm{ND}$ \\
\hline Motility & - & - & - & - & + & + & - & - & - & - & - & - & + \\
\hline Oxidase & - & - & + & $-*$ & - & + & - & $\mathrm{w}$ & $-*$ & - & - & + & + \\
\hline Nitrate reduction & - & - & + & - & + & + & - & + & + & + & - & - & - \\
\hline \multicolumn{14}{|l|}{ Decomposition of: } \\
\hline Starch & $\mathrm{W}$ & $\mathrm{W}$ & - & - & + & - & - & - & $\mathrm{w}^{\dagger}$ & + & - & - & + \\
\hline Tween 80 & + & - & + & + & - & + & + & + & + & + & + & + & + \\
\hline L-Tyrosine & + & - & + & - & + & - & - & + & + & + & - & - & + \\
\hline Xanthine & - & - & - & - & + & - & - & - & - & $\mathrm{w}$ & - & - & - \\
\hline \multicolumn{14}{|l|}{ API ZYM } \\
\hline Alkaline phosphatase & + & + & + & - & + & - & $\mathrm{w}$ & + & $\mathrm{V}$ & + & + & + & + \\
\hline Esterase $(\mathrm{C} 4)$ & + & - & + & + & - & - & + & + & $\mathrm{w}$ & $\mathrm{V}$ & $\mathrm{W}$ & - & - \\
\hline Lipase (C14) & - & - & - & $\mathrm{w}$ & - & - & - & - & - & - & - & - & - \\
\hline Valine arylamidase & + & - & - & $\mathrm{W}$ & $\mathrm{w}$ & - & + & - & $\mathrm{w}$ & + & $\mathrm{W}$ & - & + \\
\hline Cystine arylamidase & + & - & - & + & - & $\mathrm{w}$ & - & - & $\mathrm{V}$ & $\mathrm{w}$ & - & - & $\mathrm{w}$ \\
\hline Trypsin & + & - & - & - & + & + & + & - & $\mathrm{w}$ & - & + & - & + \\
\hline$\alpha$-Chymotrypsin & + & - & + & - & - & - & + & - & - & - & - & - & - \\
\hline Acid phosphatase & + & - & + & $\mathrm{w}$ & + & + & $\mathrm{w}$ & + & + & $\mathrm{w}$ & $\mathrm{w}$ & + & + \\
\hline $\begin{array}{l}\text { Naphthol-AS-BI- } \\
\text { phosphohydrolase }\end{array}$ & + & - & + & + & + & + & $\mathrm{W}$ & + & - & - & - & $\mathrm{W}$ & + \\
\hline$\alpha$-Galactosidase & - & - & - & - & - & - & - & - & - & + & - & - & - \\
\hline$\beta$-Galactosidase & - & - & $\mathrm{w}$ & - & - & - & + & - & - & + & - & - & - \\
\hline
\end{tabular}


Table 1. cont.

\begin{tabular}{|c|c|c|c|c|c|c|c|c|c|c|c|c|c|}
\hline Characteristic & 1 & 2 & 3 & 4 & 5 & 6 & 7 & 8 & 9 & 10 & 11 & 12 & 13 \\
\hline$\alpha$-Glucosidase & + & + & + & + & + & + & + & - & + & + & + & + & + \\
\hline$\beta$-Glucosidase & - & - & - & + & - & $\mathrm{w}$ & - & + & - & - & - & - & $\mathrm{w}$ \\
\hline \multicolumn{14}{|l|}{ Carbon sources utilized } \\
\hline L-Arabinose & - & - & - & - & - & - & - & $\mathrm{w}$ & - & + & - & - & - \\
\hline L-Arginine & + & $\mathrm{ND}$ & $\mathrm{ND}$ & $+^{*}$ & $-*$ & $-*$ & - & $\mathrm{ND}$ & $-*$ & - & - & ND & $\mathrm{ND}$ \\
\hline L-Asparagine & - & $\mathrm{ND}$ & $\mathrm{ND}$ & $-*$ & $t^{*}$ & $t^{*}$ & - & $\mathrm{ND}$ & $-*$ & + & + & $\mathrm{ND}$ & $\mathrm{ND}$ \\
\hline Cellobiose & + & - & + & + & + & + & + & + & - & + & $\mathrm{ND}$ & - & - \\
\hline D-Galactose & - & + & + & - & + & + & + & + & $-\dagger$ & + & + & - & - \\
\hline D-Glucose & + & - & + & + & + & + & + & + & + & + & + & + & + \\
\hline Glycerol & - & - & $\mathrm{ND}$ & $t^{*}$ & $-*$ & $-*$ & $\mathrm{w}$ & $\mathrm{ND}$ & $t^{*}$ & + & - & $\mathrm{ND}$ & - \\
\hline Inositol & - & - & - & - & + & - & - & - & $\mathrm{w} \dagger$ & - & - & ND & - \\
\hline Lactose & - & - & - & - & $+\dagger$ & + & $\mathrm{w}$ & + & - & + & - & $\mathrm{ND}$ & - \\
\hline L-Lysine & - & $\mathrm{ND}$ & $\mathrm{ND}$ & $-*$ & $-*$ & $+^{*}$ & $\mathrm{w}$ & ND & $-*$ & - & - & $\mathrm{ND}$ & $\mathrm{ND}$ \\
\hline D-Mannitol & + & - & + & - & $+\dagger$ & + & + & + & + & + & + & $\mathrm{ND}$ & - \\
\hline D-Salicin & - & - & $\mathrm{ND}$ & $t^{*}$ & $-*$ & $-*$ & - & $\mathrm{ND}$ & $-*$ & + & $\mathrm{ND}$ & - & $\mathrm{ND}$ \\
\hline Succinate & - & $\mathrm{ND}$ & $\mathrm{ND}$ & $t^{*}$ & $t^{*}$ & $t^{*}$ & + & $\mathrm{ND}$ & $t^{*}$ & + & + & + & + \\
\hline Sucrose & + & - & + & + & + & + & + & + & + & + & + & + & + \\
\hline Trehalose & + & $\mathrm{ND}$ & + & + & + & + & $\mathrm{ND}$ & + & w & $\mathrm{ND}$ & + & + & + \\
\hline D-Xylose & - & + & - & + & + & + & + & + & $\mathrm{w} \ddagger$ & + & + & - & + \\
\hline $\begin{array}{l}\text { DNA G +C content } \\
(\mathrm{mol} \%)\end{array}$ & 73 & 73 & 74 & 69 & 73 & 73 & 70 & 75 & 69 & 72 & 73 & 72 & 71 \\
\hline Isolation source & $\begin{array}{l}\text { Zooplankton- } \\
\text { enriched } \\
\text { seawater }\end{array}$ & $\begin{array}{r}\text { Beach } \\
\text { sand }\end{array}$ & $\begin{array}{l}\text { Alkaline } \\
\text { soil }\end{array}$ & Herbage & $\begin{array}{c}\text { Oil-shale } \\
\text { column }\end{array}$ & $\begin{array}{l}\text { Ground- } \\
\text { water }\end{array}$ & Tidal flat & $\begin{array}{l}\text { Alkaline } \\
\text { soil }\end{array}$ & $\begin{array}{r}\text { Saline lake, } \\
\text { Antarctica }\end{array}$ & Tidal flat & Seawater & Soil & $\begin{array}{c}\text { Industrial } \\
\text { waste- } \\
\text { water }\end{array}$ \\
\hline
\end{tabular}

${ }^{\star}$ Data from Yi \& Chun (2004a).

$\dagger$ Negative results were reported by Yi \& Chun (2004b).

$\ddagger$ Positive results were reported by Yi \& Chun (2004b). 
Culture Center of Microorganisms by using HPLC analysis (Tamaoka \& Komagata, 1984).

Cells were Gram-positive, non-motile and strictly aerobic. During the exponential growth phase, cells of strain CL-Z59 ${ }^{\mathrm{T}}$ were rods $(0.3-0.6 \mu \mathrm{m}$ in width and 0.6$1.6 \mu \mathrm{m}$ in length). Colonies on MA were circular, smooth, entire, convex and creamy white. Cells were positive for catalase activity and negative for oxidase and nitrate reductase. Cells were positive for the decomposition of casein, starch, Tween 80 and L-tyrosine but were negative for the decomposition of hypoxanthine and xanthine (Table 1).
Growth of strain CL-Z59 $9^{\mathrm{T}}$ was observed at temperatures in the range $10-35{ }^{\circ} \mathrm{C}$, the optimum being $25-$ $30{ }^{\circ} \mathrm{C}$. Growth occurred at $\mathrm{pH} 6-10$, the optimum $\mathrm{pH}$ being 6-7. The strain required $\mathrm{NaCl}$ for growth (1$10 \%, w / v)$, the optimum concentration being $3 \%$. The other results of morphological, physiological and biochemical tests are given in the species description and in Table 1.

Strain CL-Z59 ${ }^{\mathrm{T}}$ contained a tetrahydrogenated menaquinone with eight isoprene units $\left[\mathrm{MK}-8\left(\mathrm{H}_{4}\right)\right]$ as the major menaquinone, like most Nocardioides species (Holt et al., 1994) and LL-diaminopimelic acid was the

Table 2. Cellular fatty acid composition (\%) of strain CL-Z59 ${ }^{\top}$ and phylogenetically related Nocardioides species

Strains: 1, strain CL-Z59 ${ }^{\mathrm{T}} ; 2$, N. marinisabuli SBS-12 ${ }^{\mathrm{T}} ; 3$, N. kribbensis KSL-2 ${ }^{\mathrm{T}} ; 4$, N. plantarum DSM $11054^{\mathrm{T}} ; 5$, N. pyridinolyticus OS4 ${ }^{\mathrm{T}} ; 6, N$. aquiterrae GW-9 ${ }^{\mathrm{T}}$; 7, N. aestuarii JC2056 ${ }^{\mathrm{T}}$; 8, N. lentus KSL-17 ${ }^{\mathrm{T}}$; 9, N. aquaticus EL-17K ${ }^{\mathrm{T}} ; 10$, N. ganghwensis JC2055 ${ }^{\mathrm{T}}$; 11, N. marinus CL-DD14 ${ }^{\mathrm{T}}$; 12, N. kongjuensis A2-4 ${ }^{\mathrm{T}} ; 13$, N. nitrophenolicus NSP41 ${ }^{\mathrm{T}}$. Data are from Yoon et al. (1997, 1999, 2004, 2005b, 2006a, b), Lawson et al. (2000), Yi \& Chun (2004a, b), Choi et al. (2007) and Lee et al. (2007). Fatty acids that represent $<0.5 \%$ in all strains have been omitted. -, Not detected or $<0.1 \%$. Double bond position indicated by a capital letter is unknown (Yoon et al., 2004).

\begin{tabular}{|c|c|c|c|c|c|c|c|c|c|c|c|c|c|}
\hline Fatty acid & 1 & 2 & 3 & 4 & 5 & 6 & 7 & 8 & 9 & 10 & 11 & 12 & 13 \\
\hline \multicolumn{14}{|l|}{ Saturated } \\
\hline $\mathrm{C}_{10: 0}$ & - & - & - & 5.7 & - & - & 1.4 & - & - & 1.6 & - & - & - \\
\hline $\mathrm{C}_{15: 0}$ & - & - & - & 1.2 & 0.4 & 0.4 & - & 1.0 & 2.4 & 1.1 & 0.3 & - & - \\
\hline $\mathrm{C}_{16: 0}$ & 1.0 & 2.7 & 3.6 & 10.5 & 1.2 & 0.5 & - & 1.9 & 14.1 & 0.8 & 1.3 & 3.9 & 2.3 \\
\hline $\mathrm{C}_{17: 0}$ & - & 1.0 & - & 4.4 & 1.2 & 0.5 & - & 1.8 & 5.9 & 2.0 & 0.3 & 1.4 & 0.9 \\
\hline $\mathrm{C}_{18: 0}$ & - & 2.4 & 1.9 & 6.8 & 1.0 & 0.3 & - & - & 6.9 & 0.2 & 0.4 & 3.4 & - \\
\hline \multicolumn{14}{|l|}{ Unsaturated } \\
\hline $\mathrm{C}_{17: 1} \omega 6 c$ & - & - & - & 2.6 & - & - & - & 1.7 & - & 1.6 & 0.2 & 11.2 & 10.8 \\
\hline $\mathrm{C}_{17: 1} \omega 8 c$ & 0.5 & 2.8 & 2.4 & 4.4 & 1.7 & 1.7 & 7.0 & 6.6 & - & 25.7 & 3.8 & 2.9 & 1.4 \\
\hline $\mathrm{C}_{18: 1} \omega 7 c$ & 2.4 & - & 1.9 & 4.0 & - & - & - & - & - & 1.3 & 1.6 & - & - \\
\hline $\mathrm{C}_{18: 1} \omega 9 c$ & 1.4 & 5.6 & 7.2 & 5.0 & 2.0 & 0.9 & 2.7 & 3.1 & $30.6^{*}$ & 5.7 & 4.4 & 5.0 & 4.5 \\
\hline \multicolumn{14}{|l|}{ Branched } \\
\hline iso- $\mathrm{C}_{12: 0}$ & 0.3 & - & - & 2.0 & - & - & - & - & - & 0.6 & - & - & - \\
\hline iso- $\mathrm{C}_{14: 0}$ & 3.2 & - & 5.4 & 0.8 & 0.5 & 1.5 & 1.8 & 2.3 & - & 1.3 & 2.0 & - & 0.4 \\
\hline iso- $\mathrm{C}_{15: 0}$ & 3.4 & 3.8 & 9.7 & 2.5 & 5.1 & 4.9 & 1.2 & 0.7 & - & 2.7 & 0.3 & 2.2 & 3.1 \\
\hline anteiso- $\mathrm{C}_{15: 0}$ & 0.5 & - & 2.0 & - & 1.4 & 2.4 & 1.3 & - & - & - & 0.2 & - & 0.5 \\
\hline iso- $\mathrm{C}_{16: 1} \mathrm{H}$ & 4.6 & 1.4 & - & 0.3 & 4.2 & $6.8^{\star}$ & 14.5 & - & - & 4.4 & 0.3 & - & 1.3 \\
\hline iso- $\mathrm{C}_{16: 0}$ & 65.3 & 48.7 & 42.5 & 6.2 & 47.3 & 57.6 & 52.0 & 60.9 & 8.1 & 30.4 & 71.5 & 27.1 & 28.1 \\
\hline iso- $\mathrm{C}_{17: 0}$ & 2.5 & 10.8 & 5.7 & 2.1 & 1.7 & 0.7 & - & 1.0 & - & 2.4 & 0.6 & 6.3 & 12.3 \\
\hline anteiso- $C_{17: 0}$ & 2.3 & 4.3 & 4.2 & - & 14.2 & 9.0 & 1.8 & 1.5 & 10.6 & - & 0.7 & 3.0 & 5.8 \\
\hline iso- $\mathrm{C}_{17: 1} \omega 9 c$ & - & 2.7 & 2.3 & 0.5 & - & - & - & - & - & 1.6 & - & - & - \\
\hline anteiso- $\mathrm{C}_{17: 1} \omega 9 c$ & 0.1 & - & - & - & 2.1 & 2.4 & - & - & - & - & - & - & - \\
\hline iso- $\mathrm{C}_{18: 0}$ & 1.4 & 2.2 & 1.4 & 0.2 & 0.5 & 0.2 & - & 1.5 & 1.3 & 1.2 & 2.1 & 1.4 & 1.6 \\
\hline \multicolumn{14}{|l|}{ 10-Methyl } \\
\hline $\mathrm{C}_{16: 0}$ & 5.2 & - & - & - & 5.0 & 3.4 & 1.3 & 0.9 & - & - & 1.4 & 3.2 & 3.6 \\
\hline $\mathrm{C}_{17: 0}$ & 1.4 & 1.7 & 1.5 & 6.0 & 5.5 & 4.1 & 3.1 & 5.8 & - & 3.9 & 5.1 & 6.0 & 5.1 \\
\hline $\mathrm{C}_{18: 0}(\mathrm{TBSA}) \dagger$ & 0.5 & - & 1.2 & 8.4 & 3.4 & 0.8 & 0.3 & 1.6 & 3.6 & - & 1.7 & 14.4 & 13.9 \\
\hline \multicolumn{14}{|l|}{ Hydroxy } \\
\hline $\mathrm{C}_{17: 0} 3-\mathrm{OH}$ & 0.7 & - & - & - & - & - & - & 2.7 & - & - & - & - & - \\
\hline Summed feature $3 \ddagger$ & 3.2 & 2.9 & 3.1 & 0.6 & 0.8 & 0.7 & 1.3 & 1.8 & - & 1.9 & 1.3 & 2.3 & - \\
\hline
\end{tabular}

${ }^{\star} \mathrm{C}_{18: 1}$ (Lawson et al., 2000).

$\dagger$ TBSA, Tuberculostearic acid.

$\ddagger$ Summed feature 3 contains iso- $\mathrm{C}_{15: 0} 2-\mathrm{OH}$ and/or $\mathrm{C}_{16: 1} \omega 7 c$. 
cell-wall diamino acid, as in Nocardioides species (Prauser, 1976). The fatty acid profile for strain CL$\mathrm{Z} 9^{\mathrm{T}}$ was dominated by iso- $\mathrm{C}_{16: 0} \quad(65.3 \%$; Table 2$)$, which is typically found as a major component in members of the genus Nocardioides (Yoon et al., 1999). The DNA G+C content of strain CL-Z59 ${ }^{\mathrm{T}}$ was $73.3 \mathrm{~mol} \%$, being within the range $(67-75 \mathrm{~mol} \%$;
Prauser, 1976; Yoon et al., 2006a) reported for recognized species of the genus Nocardioides.

Phylogenetic trees based on 16S rRNA gene sequences showed that strain CL-Z59 ${ }^{\mathrm{T}}$ falls within the radiation of the cluster comprising Nocardioides species (Fig. 1). Sequence similarity indicated that the closest relative of

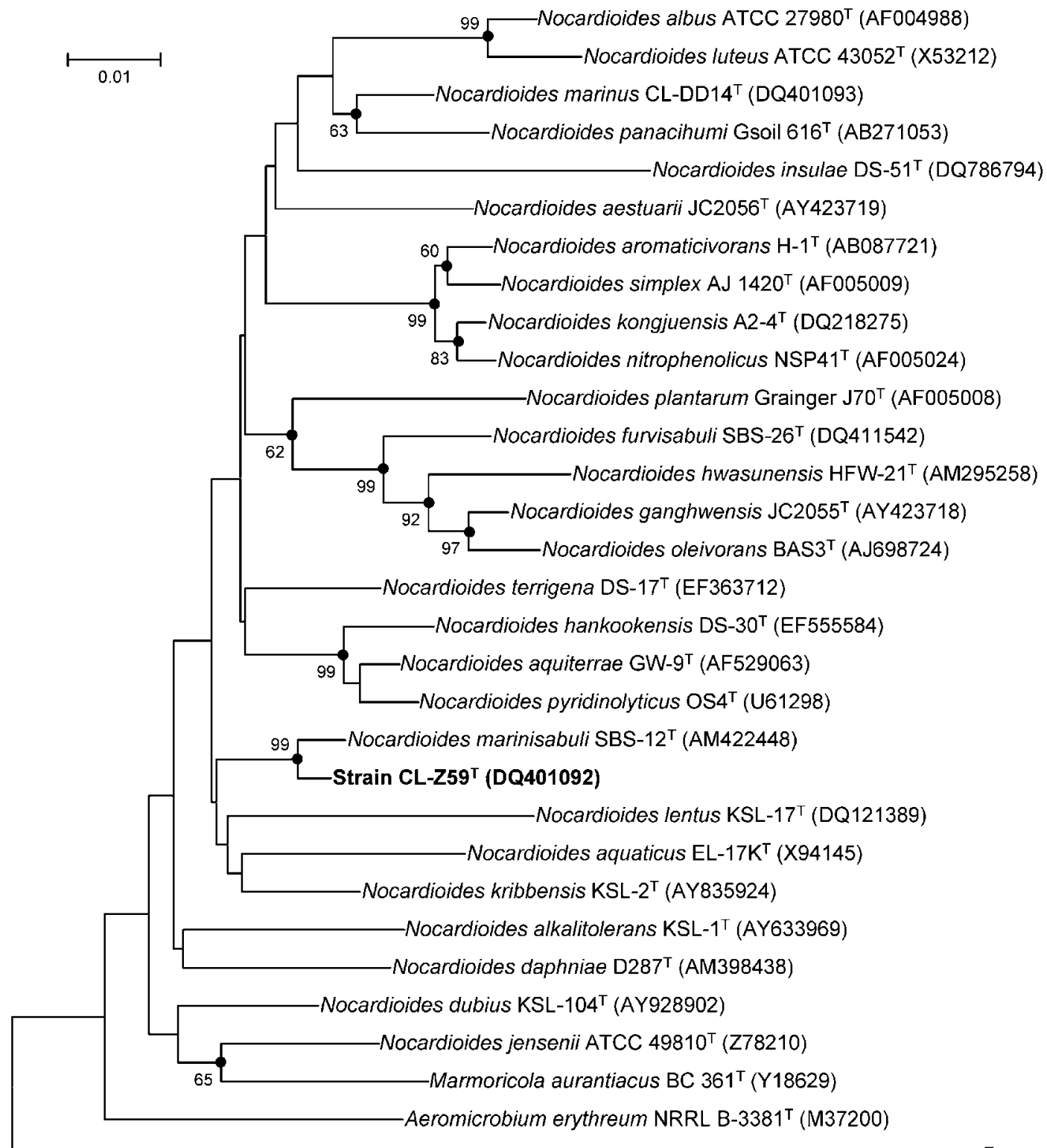

Terrabacter tumescens ATCC $6947^{\top}$ (AF005023)

Fig. 1. Neighbour-joining phylogenetic tree, based on 16S rRNA gene sequences, showing the relationships between strain $\mathrm{CL}-Z 59^{\top}$ and members of the genus Nocardioides. Bootstrap percentages (based on 1000 replications) greater than $60 \%$ are shown at nodes. Accession numbers are given in parentheses. Filled circles indicate that the corresponding nodes were also obtained in the maximum-parsimony tree. Terrabacter tumescens ATCC $6947^{\top}$ (AF005023) was used as an outgroup. Bar, 0.01 nucleotide substitutions per site. 
strain CL-Z59 ${ }^{\mathrm{T}}$ was N. marinisabuli $(99.2 \%)$; the other members of the genus Nocardioides showed sequence similarities in the range $94.2-97.1 \%$. In all of the phylogenetic trees, strain CL-Z59 ${ }^{\mathrm{T}}$ formed a tight clade with $N$. marinisabuli SBS- $12^{\mathrm{T}}$ (Fig. 1). This clade was related to the nearest clade, containing N. kribbensis, $N$. aquaticus and N. lentus (Fig. 1). The levels of DNA-DNA relatedness between strain $\mathrm{CL}-\mathrm{Z} 59^{\mathrm{T}}$ and $N$. marinisabuli KCCM $42681^{\mathrm{T}}$ and between strain CL-Z59 ${ }^{\mathrm{T}}$ and $N$. kribbensis KSL- ${ }^{\mathrm{T}}$ were $33 \pm 9 \%$ and $18 \pm 6 \%$, respectively, supporting the view that strain CL-Z5 $59^{\mathrm{T}}$ is a distinct species (Stackebrandt \& Goebel, 1994). Furthermore, strain CL-Z59 ${ }^{\mathrm{T}}$ can be clearly distinguished from $N$. marinisabuli on the basis of phenotypic traits: $\mathrm{pH}$, temperature and $\mathrm{NaCl}$ range for growth, the ability to decompose Tween 80 and L-tyrosine, the ability to produce acid phosphatase, $\alpha$-chymotrypsin, cystine arylamidase, esterase (C4), naphthol-AS-BI-phosphohydrolase, trypsin and valine arylamidase and the utilization of cellobiose, citrate, D-fructose, D-galactose, D-glucose, Dmannitol, D-ribose, sucrose and D-xylose (Table 1). In addition, phenotypic, biochemical and chemotaxonomic features, including $\mathrm{NaCl}$ ranges for growth, optimal $\mathrm{pH}$, the absence of oxidase and nitrate reductase, the carbonutilization pattern and the fatty acid profiles, clearly served to differentiate strain CL-Z59 ${ }^{\mathrm{T}}$ from N. kribbensis (Tables 1 and 2). Notably, combinations of some phenotypic traits (e.g. alkaline phosphatase, $\alpha$-chymotrypsin, $\alpha$-galactosidase, $\alpha$-glucosidase and utilization of Larabinose, cellobiose, D-glucose, inositol, D-mannitol, succinate and D-xylose) can serve to differentiate species of the genus Nocardioides (Table 1). In conclusion, based on the evidence from the polyphasic study, strain CL$\mathrm{Z} 59^{\mathrm{T}}$ represents a novel species of the genus Nocardioides, for which the name Nocardioides salarius sp. nov. is proposed.

\section{Description of Nocardioides salarius sp. nov.}

Nocardioides salarius (sa.la' ri.us. L. masc. adj. salarius of, or belonging to, salt, referring to the isolation of this microorganism from seawater).

Cells are Gram-positive, non-motile, strictly aerobic rods approximately $0.6-1.6 \mu \mathrm{m}$ long and $0.3-0.6 \mu \mathrm{m}$ wide. Colonies on MA are creamy white and circular, smooth and convex with entire margins. Growth occurs at 10$35{ }^{\circ} \mathrm{C}$ (optimally at $25-30{ }^{\circ} \mathrm{C}$ ) and at $\mathrm{pH}$ 6-10 (optimally at $\mathrm{pH} 6-7)$. Growth occurs at $\mathrm{NaCl}$ concentrations of 1$10 \%(\mathrm{w} / \mathrm{v})$ (optimally at $3 \%$ ). Positive for catalase but negative for oxidase and nitrate reductase. Casein, starch, Tween 80 and L-tyrosine are hydrolysed but hypoxanthine and xanthine are not. In the API 20NE system, aesculin hydrolysis and gelatinase are positive but nitrate reduction, indole production, glucose fermentation, arginine dihydrolase, urease and $\beta$-galactosidase are negative. In the API ZYM system, acid phosphatase, alkaline phosphatase, $\alpha$-chymotrypsin, cystine arylamidase, esterase
(C4), esterase lipase (C8), $\alpha$-glucosidase, leucine arylamidase, trypsin, naphthol-AS-BI-phosphohydrolase and valine arylamidase are positive but $N$-acetyl- $\beta$-glucosaminidase, $\alpha$-fucosidase, $\alpha$-galactosidase, $\beta$-galactosidase, $\beta$ glucosidase, $\beta$-glucuronidase, lipase (C14) and $\alpha$-mannosidase are negative. Utilizes L-arginine, cellobiose, Dglucose, D-mannitol, L-ornithine, D-ribose, sucrose and trehalose, but not $\mathrm{N}$-acetylglucosamine, L-arabinose, Lasparagine, citrate, D-fructose, D-galactose, glycerol, inositol, lactose, L-lysine, D-mannose, raffinose, L-rhamnose, D-salicin, succinate and D-xylose as sole carbon sources. The major menaquinone is $\mathrm{MK}-8\left(\mathrm{H}_{4}\right)$. The cell-wall diamino acid is LL-diaminopimelic acid. The major fatty acid is iso- $\mathrm{C}_{16: 0}(65.3 \%)$. The DNA G $+\mathrm{C}$ content of the type strain is $73.3 \mathrm{~mol} \%$.

The type strain, CL-Z59 ${ }^{\mathrm{T}} \quad\left(=\mathrm{KCCM} \quad 42320^{\mathrm{T}}=\mathrm{DSM}\right.$ $\left.18239^{\mathrm{T}}\right)$, was isolated from seawater enriched with zooplankton.

\section{Acknowledgements}

We thank Dr J. H. Yoon (KRIBB) for discussions during this work. We also acknowledge the expert technical support of Ms In-Sung Lee (electron microscopy) of the National Center for Inter-University Research Facilities at Seoul National University. This work was supported by the BK21 project of the Korean Government and the Ministry of Maritime Affairs and Fisheries (the Korea EAST-1 program).

\section{References}

Altschul, S. F., Gish, W., Miller, W., Myers, E. W. \& Lipman, D. J. (1990). Basic local alignment search tool. J Mol Biol 215, $403-410$.

An, D.-S., Im, W.-T., Lee, S.-T. \& Yoon, M.-H. (2007). Nocardioides panacihumi sp. nov., isolated from soil of a ginseng field. Int J Syst Evol Microbiol 57, 2143-2146.

Cho, J. C. \& Giovannoni, S. J. (2004). Cultivation and growth characteristics of a diverse group of oligotrophic marine Gammaproteobacteria. Appl Environ Microbiol 70, 432-440.

Choi, D. H., Kim, H. M., Noh, J.-H. \& Cho, B. C. (2007). Nocardioides marinus sp. nov. Int J Syst Evol Microbiol 57, 775-779.

Cole, J. R., Chai, B., Marsh, T. L., Farris, R. J., Wang, Q., Kulam, S. A., Chandra, S., McGarrell, D. M., Schmidt, T. M. \& other authors (2003). The Ribosomal Database Project (RDP-1l): previewing a new autoaligner that allows regular updates and the new prokaryotic taxonomy. Nucleic Acids Res 31, 442-443.

Collins, M. D. (1985). Analysis of isoprenoid quinones. Methods Microbiol 18, 329-366.

Collins, M. D., Dorsch, M. \& Stackebrandt, E. (1989). Transfer of Pimelobacter tumescens to Terrabacter gen. nov. as Terrabacter tumescens comb. nov. and of Pimelobacter jensenii to Nocardioides as Nocardioides jensenii comb. nov. Int J Syst Bacteriol 39, 1-6.

Collins, M. D., Cockcroft, S. \& Wallbanks, S. (1994). Phylogenetic analysis of a new LL-diaminopimelic acid-containing coryneform bacterium from herbage, Nocardioides plantarum sp. nov. Int J Syst Bacteriol 44, 523-526. 
Englen, M. D. \& Kelley, L. C. (2000). A rapid DNA isolation procedure for the identification of Campylobacter jejuni by the polymerase chain reaction. Lett Appl Microbiol 31, 421-426.

Fitch, W. M. (1971). Toward defining the course of evolution: minimum change for a specific tree topology. Syst Zool 20, 406-416.

Hansen, G. H. \& Sørheim, R. (1991). Improved method for phenotypical characterization of marine bacteria. $J$ Microbiol Methods 13, 231-241.

Holt, J. G., Krieg, N. R., Sneath, P. H. A., Staley, J. T. \& Williams, S. T. (1994). Nocardioform actinomycetes. In Shorter Bergey's Manual of Determinative Bacteriology, pp. 625-650. Edited by W. R. Hensyl. Baltimore: Williams \& Wilkins.

Jensen, H. L. (1934). Studies on saprophytic mycobacteria and corynebacteria. Proc Linn Soc N S W 59, 19-61.

Jeon, Y.-S., Chung, H., Park, S., Hur, I., Lee, J.-H. \& Chun, J. (2005). jPHYDIT: a JAVA-based integrated environment for molecular phylogeny of ribosomal RNA sequences. Bioinformatics 21, 31713173 .

Jukes, T. H. \& Cantor, C. R. (1969). Evolution of protein molecules. In Mammalian Protein Metabolism, pp. 21-132. Edited by H. N. Munro. New York: Academic Press.

Kim, Y.-G., Choi, D. H., Hyun, S. \& Cho, B. C. (2007). Oceanobacillus profundus sp. nov., isolated from a deep-sea sediment core. Int J Syst Evol Microbiol 57, 409-413.

Kubota, M., Kawahara, K., Sekiya, K., Uchida, T., Hattori, Y., Futamata, H. \& Hiraishi, A. (2005). Nocardioides aromaticivorans $\mathrm{sp.}$ nov., a dibenzofuran-degrading bacterium isolated from dioxinpolluted environments. Syst Appl Microbiol 28, 165-174.

Kumar, S., Tamura, K. \& Nei, M. (2004). MEGA3: integrated software for molecular evolutionary genetics analysis and sequence alignment. Brief Bioinform 5, 150-163.

Lane, D. J. (1991). 16S/23S rRNA sequencing. In Nucleic Acid Techniques in Bacterial Systematics, pp. 115-175. Edited by E. Stackebrandt \& M. Goodfellow. Chichester: Wiley.

Lawson, P. A., Collins, M. D., Schumann, P., Tindall, B. J., Hirsch, P. \& Labrenz, M. (2000). New LL-diaminopimelic acid-containing actinomycetes from hypersaline, heliothermal and meromictic Antarctic Ekho Lake: Nocardioides aquaticus sp. nov. and Friedmanniella lacustris sp. nov. Syst Appl Microbiol 23, 219-229.

Lee, S. D. (2007). Nocardioides furvisabuli sp. nov., isolated from black sand. Int J Syst Evol Microbiol 57, 35-39.

Lee, D. W., Hyun, C.-G. \& Lee, S. D. (2007). Nocardioides marinisabuli sp. nov., a novel actinobacterium isolated from beach sand. Int J Syst Evol Microbiol 57, 2960-2963.

Lee, S. D., Lee, D. W. \& Kim, J.-S. (2008). Nocardioides hwasunensis sp. nov. Int J Syst Evol Microbiol 58, 278-281.

Lemos, M. L., Toranzo, A. E. \& Barja, J. L. (1985). Modified medium for the oxidation-fermentation test in the identification of marine bacteria. Appl Environ Microbiol 49, 1541-1543.

Lyman, J. \& Fleming, R. H. (1940). Composition of sea water. J Mar Res 3, 134-146.

Minnikin, D. E., O'Donnell, A. G., Goodfellow, M., Alderson, G., Athalye, M., Schaal, A. \& Parlett, J. H. (1984). An integrated procedure for the extraction of bacterial isoprenoid quinones and polar lipids. J Microbiol Methods 2, 233-241.

Prauser, H. (1976). Nocardioides, a new genus of the order Actinomycetales. Int J Syst Bacteriol 26, 58-65.

Prauser, H. (1984). Nocardioides luteus spec. nov. Z Allg Mikrobiol 24, 647-648.
Saitou, N. \& Nei, M. (1987). The neighbor-joining method: a new method for reconstructing phylogenetic trees. Mol Biol Evol 4, 406-425.

Schippers, A., Schumann, P. \& Spröer, C. (2005). Nocardioides oleivorans sp. nov., a novel crude-oil-degrading bacterium. Int J Syst Evol Microbiol 55, 1501-1504.

Smibert, R. M. \& Krieg, N. R. (1994). Phenotypic characterization. In Methods for General and Molecular Bacteriology, pp. 607-654. Edited by P. Gerhardt, R. G. E. Murray, W. A. Wood \& N. R. Krieg. Washington, DC: American Society for Microbiology.

Stackebrandt, E. \& Goebel, B. M. (1994). Taxonomic note: a place for DNA-DNA reassociation and $16 \mathrm{~S}$ rRNA sequence analysis in the present species definition in bacteriology. Int J Syst Bacteriol 44, 846-849.

Staneck, J. L. \& Roberts, G. D. (1974). Simplified approach to the identification of aerobic actinomycetes by thin-layer chromatography. Appl Microbiol 28, 226-231.

Suzuki, K. \& Komagata, K. (1983). Pimelobacter gen. nov., a new genus of coryneform bacteria with LL-diaminopimelic acid in the cell wall. J Gen Appl Microbiol 29, 59-71.

Tamaoka, J. \& Komagata, K. (1984). Determination of DNA base composition by reversed-phase high-performance liquid chromatography. FEMS Microbiol Lett 25, 125-128.

Tóth, E. M., Kéki, Z., Homonnay, Z. G., Borsodi, A. K., Márialigeti, K. \& Schumann, P. (2008). Nocardioides daphniae sp. nov., isolated from Daphnia cucullata (Crustacea: Cladocera). Int J Syst Evol Microbiol 58, 78-83.

Yi, H. \& Chun, J. (2004a). Nocardioides aestuarii sp. nov., isolated from tidal flat sediment. Int J Syst Evol Microbiol 54, 2151-2154.

Yi, H. \& Chun, J. (2004b). Nocardioides ganghwensis sp. nov., isolated from tidal flat sediment. Int J Syst Evol Microbiol 54, 1295-1299.

Yoon, J.-H., Rhee, S.-K., Lee, J.-S., Park, Y.-H. \& Lee, S.-T. (1997). Nocardioides pyridinolyticus sp. nov., a pyridine-degrading bacterium isolated from the oxic zone of an oil shale column. Int J Syst Bacteriol 47, 933-938.

Yoon, J.-H., Cho, Y.-G., Lee, S. T., Suzuki, K.-I., Nakase, T. \& Park, Y.-H. (1999). Nocardioides nitrophenolicus sp. nov., a p-nitrophenoldegrading bacterium. Int J Syst Bacteriol 49, 675-680.

Yoon, J.-H., Kim, I.-G., Kang, K. H., Oh, T.-K. \& Park, Y.-H. (2004). Nocardioides aquiterrae sp. nov., isolated from groundwater in Korea. Int J Syst Evol Microbiol 54, 71-75.

Yoon, J.-H., Kim, I.-G., Lee, M.-H., Lee, C.-H. \& Oh, T.-K. (2005a). Nocardioides alkalitolerans sp. nov., isolated from an alkaline serpentinite soil in Korea. Int J Syst Evol Microbiol 55, 809-814.

Yoon, J.-H., Kim, I.-G., Lee, M.-H. \& Oh, T.-K. (2005b). Nocardioides kribbensis sp. nov., isolated from an alkaline soil. Int J Syst Evol Microbiol 55, 1611-1614.

Yoon, J.-H., Lee, C.-H. \& Oh, T.-K. (2005c). Nocardioides dubius sp. nov., isolated from an alkaline soil. Int J Syst Evol Microbiol 55, 2209-2212.

Yoon, J.-H., Lee, C.-H. \& Oh, T.-K. (2006a). Nocardioides lentus sp. nov., isolated from an alkaline soil. Int J Syst Evol Microbiol 56, 271-275.

Yoon, J.-H., Lee, J.-K., Jung, S.-Y., Kim, J.-A., Kim, H.-K. \& Oh, T.-K. (2006b). Nocardioides kongjuensis sp. nov., an $N$-acylhomoserine lactone-degrading bacterium. Int J Syst Evol Microbiol 56, 1783-1787.

Yoon, J.-H., Kang, S.-J., Lee, C.-H. \& Oh, T.-K. (2007a). Nocardioides insulae sp. nov., isolated from soil. Int J Syst Evol Microbiol 57, 136-140. 
Yoon, J.-H., Kang, S.-J., Lee, S.-Y. \& Oh, T.-K. (2007b). Nocardioides terrigena sp. nov., isolated from soil. Int J Syst Evol Microbiol 57, 2472-2475.
Yoon, J.-H., Kang, S.-J., Lee, M.-H. \& Oh, T.-K. (2008). Nocardioides hankookensis sp. nov., isolated from soil. Int J Syst Evol Microbiol 58, 434-437. 